\section{Welche Stadium-I-Seminome rezidivieren nach adjuvanter Carboplatin-Therapie?}

\author{
Therapieoptionen für Stadium-I-Seminome sind nach einer inguinalen \\ Orchiektomie kontrollierendes Abwarten oder eine adjuvante Strahlen- \\ bzw. Chemotherapie. In einer Langzeitstudie wurden Prognosemarker für \\ Rezidive nach einer Carboplatin-Therapie gesucht.
}

gnome-induzierenden Radiotherapie bevorzugt.

Eine retrospektive Untersuchung von 517 zwischen 1996 und 2013 in Großbritannien behandelten Patienten sollte nun zeigen, welche Faktoren für ein Rezidiv nach Carboplatin-Therapie prädestinieren. Innerhalb eines medianen Follow-ups von 47,2 Monaten (0,4-214) errechnete sich eine Rate für das rezidivfreie Überleben von $95 \%$ (95\%-Konfidenzintervall 92,8-97,3). Die mediane Zeit bis zum Rückfall betrug 22,7 Monate $(12,5-109,5)$, wobei Rezidive nach 3 Jahren mit 0,8\% sehr selten vorkamen. 21 Patienten entwickelten ein Rezidiv. Bei 20 von ihnen $(95 \%)$ waren retroperitoneale Lymphknotenmetastasen nachweisbar; $16(76 \%)$ hatten zum Rezidivzeitpunkt erhöhte Tumormarker wie $\beta$-humanes Choriongonadotropin ( $\beta$-HCG) und/ oder Lactatdehydrogenase (LDH). In der multivariaten Analyse erwies sich die Tumorgröße als die einzige signifikant rezidivassoziierte Variable.

Fazit: Patienten mit Stadium-I-Seminom haben nach inguinaler Orchiektomie und adjuvanter Einzeldosis-Carboplatin-Therapie mit einer Rezidivrate von $5 \%$ eine gute Prognose. Rezidive, überwiegend des kontralateralen Hodens, treten praktisch ausschließlich in den ersten 2 Jahren postoperativ auf. Prognostisch bedeutsam ist laut einer retrospektiven Analyse womöglich die Tumorgröße.

Barbara Kreutzkamp

Chau $C$ et al. Treatment outcome and patterns of relapse following adjuvant carboplatin for stage I testicular seminomatous germ-cell tumor: results from a 17-year UK experience. Ann Oncol. 2015;26(9):1865-70.

\title{
Bestrahlung plus ADT bei lokal fortgeschrittenem Prostatakarzinom nur vorübergehend belastend
}

\section{In der Phase-III-Studie NCIC CTG PR3/MRC PR07 lebten Patienten mit lokal fortgeschrittenem Prostatakarzinom unter der Kombination Strahlentherapie plus Androgendeprivation (ADT) deutlich länger als unter einer alleinigen lebenslangen ADT. Die Frage war jetzt, ob dieser Vorteil mit einer deutlich schlechteren Lebensqualität erkauft werden muss.}

$B_{s}$ ereits 2011 wurden die Ergebnisse der Studie mit 1.205 Patienten zum Stellenwert der Strahlentherapie zusätzlich zur ADT veröffentlicht [Warde $P$ et al. Lancet. 2011;378(9809):2104-11]. Die Patienten hatten ein lokal fortgeschrittenes T3- oder T4- oder ein T2-Prostatakarzinom mit Risikofaktoren. Im Vergleich zur alleinigen Androgendeprivation führte die zusätzliche Strahlentherapie zu einem signifikant besseren Überleben, die 7-Jahres-Überlebensrate stieg von 66 auf $74 \%$ (Hazard Ratio 0,77; p = 0,033).

Nun wurde über die Langzeitergebnisse zur gesundheitsbezogenen Lebensqualität (HR-QOL) berichtet. Diese wurden als „patient reported outcomes“ er- fasst. Hierzu füllten die Patienten den Fragebogen EORTC QLQ-C30 plus eine zusätzliche Checkliste für prostataspezifische Aspekte aus oder den FACT-P (Functional Assessment of Cancer Therapy-Prostate questionnaire). Die Lebensqualität wurde zu Beginn der Studie, am Bestrahlungsende, in den ersten 2 Jahren alle 6 Monate und danach jährlich bestimmt.

In beiden Studienarmen war der Rücklauf der Fragebögen sehr hoch. Die Strahlentherapie führte $\mathrm{zu}$ vorübergehenden lokoregionären Toxizitäten. So kam es nach 6 Monaten signifikant häufiger zu Darmbeschwerden ( $p=0,02)$, Durchfällen ( $p<0,001)$, Blasenfunkti- onsstörungen ( $\mathrm{p}=0,003)$ und zur erektilen Dysfunktion ( $\mathrm{p}=0,008)$. Diese Symptome bildeten sich jedoch zurück, sodass nach 36 Monaten und auch später keine Unterschiede mehr zwischen den Studienarmen nachweisbar waren. In beiden Gruppen nahm die Lebensqualität jedoch im Laufe der Jahre gleichartig ab. Diese Verschlechterung war im FACT-PFragebogen sowie dem EORTC QLQC30 zu messen (jeweils $\mathrm{p}<0,001$ ). Zwischen den Therapiearmen konnten dagegen im weiteren Verlauf keine Unterschiede mehr nachgewiesen werden.

Fazit: Bei Patienten mit lokal fortgeschrittenem Prostatakarzinom verbessert die Bestrahlung zusätzlich zur ADT das Überleben signifikant und hat nur einen mäßigen und vorübergehenden negativen Einfluss auf die Lebensqualität.

Brigitte Schalhorn

Brundage $M$ et al. Impact of radiotherapy when added to androgen-deprivation therapy for locally advanced prostate cancer: long-term quality-of-life outcomes from the NCIC CTG PR3/MRC PR07 randomized trial. J Clin Oncol. 2015;33(19):2151-7. 